\title{
On the effect of the microstructure on the dynamic behaviour of Ti-6Al-4V
}

\author{
Sergio Perosanz ${ }^{1 *}$, Manuel Viscasillas ${ }^{1}$, Nuria Martin Piris ${ }^{1}$, Mikko Hokka ${ }^{2}$, and Daniel \\ Barba $^{1}$ \\ ${ }^{1}$ Escuela Técnica Superior de Ingeniería Aeronáutica y del Espacio, Universidad Politécnica de \\ Madrid, Plaza Cardenal Cisneros 3, 28040 Madrid, Spain. \\ 2 Impact - Multiscale Mechanics Research Group, Engineering Materials Science, Tampere \\ University, POB 589, FI-33014, Tampere, Finland
}

\begin{abstract}
Aerospace components in jet engines need to outstand extreme conditions of high-temperatures and loads. Moreover, these components can sometimes undergo dynamic conditions if impact occurs during the flight. It is critical to understand the behaviour of aerospace alloys under these combined extreme conditions of high-temperature and dynamics loads. One of the most extended alloys used in the compressor and fan stages of commercial jet engines is Ti-6Al4V. The dynamic properties of Ti-6Al-4V are strongly dependent on the microstructure state and the temperature conditions. However, these dependencies are yet not fully understood. Moreover, this alloy can present a wide variety of microstructures depending on the component and manufacturing methods. In this work, we compare the response of five typical Ti-6Al-4V microstructures tested under static and dynamic conditions and different temperatures. The macroscopic response of the alloy is rationalised on the basis of its microstructural state using combined microscopy characterisation and computational modelling. To this end, computational plastic models are constructed and validated against experimental observations. In this way, the relationship between the mechanical properties of each microstructure and the temperature and strain rate conditions can be extracted to optimize the material state under specific dynamics in-service conditions.
\end{abstract}

\section{Introduction}

The aerospace industry requires materials with great resistance, reliability, and good behaviour against corrosion and oxidation, but which at the same time are as light as possible. Weight is the most limiting factor in aircraft design, greatly influencing the entire cost cycle. In this regard, titanium alloys arise as the perfect candidate to meet these needs, being widely used in the aerospace industry since the development of the Kroll obtention process in the 40 s.

Among the existing titanium alloys, Ti-6l-4V is one of the most studied and technologically developed alloys in the industry leading to high use rates in aerospace

* Sergio Perosanz: sergio.perosanz.amarillo@alumnos.upm.es 
components. Although being far from being the titanium alloy with the best mechanical properties of all those available, but its good combination of properties and its versatility in their mechanical properties through the application of different heat treatments, makes it a preferable choice for structural components in jet-engine engines.

These components can be subjected to a wide variety of extreme temperature and loading conditions. Among them, dynamics conditions during service can occur in an event of impact, so it is vital to understand the behaviour of this alloy under dynamic loads in combination of high temperatures. Furthermore, the mechanical properties have a strong dependence on the microstructure as mentioned before and this alloy presents a great variety depending on the component and the manufacturing methods.[1]

Strong efforts have been done to address the mechanical properties of Ti-6Al-4V under a variety of temperature and strain rate conditions [2-6]. The work of Lee et al. [2] present a complete study of the dynamic compressive mechanical behaviour of Ti-6Al-4V under a wide range of temperatures $\left(20^{\circ} \mathrm{C}\right.$ to $\left.1100^{\circ} \mathrm{C}\right)$. They found adiabatic shear banding the main failure mode in this range of strain rate and temperature conditions. However, no effect of microstructure was study. More recently, the work of Lee et al. [3] present a study in which the effect of microstructural features in Ti-6Al-4V under dynamics torsional conditions was addressed. They found that coarse Widmanstätten microstructure were more prone to adiabatic shear band deformation than thinner microstructure. This study was restricted to lamellar $\alpha$ structures. Gangireddy et al. [4] study the effect of rapid heating in the dynamic behaviour of Ti-6Al-4V, focusing on the phase transformation appearing above $800^{\circ} \mathrm{C}$. They rationalised the thermal softening behaviour using Johnson-Cook viscoplastic constitutive equations. Lin et al. [5] studied the microstructural changes during dynamic tensile deformation of Ti-6Al-4V in lamellar form. They found a stress softening associated with globurarisation of the $\alpha$ case. In this case, they used an Arrhenius-type equation combine with Hensel-Spittle model to rationalise the dynamic and microstructural evolution observed experimentally. In a recent work, Hueto et al. [6] studied the effect of temperature on the dynamic behaviour of Ti-6Al-4V following two heating methods: electric heating method by Joule effect and conventional furnace heating. The results were rationalised using JohnsonCook constitutive models. Although these studies provide insight of the dynamic behaviour of Ti-6Al-4V under different conditions, further research is needed in order to understand the effect of microstructure on the dynamic behaviour of this alloy. These required efforts need to combine a carefully designed systematic experimental and characterisation of the relevant microstructures for Ti-6Al-4V under static and dynamics conditions with advanced computational tools to gain insights of the observed behaviour.

To fill this gap, the responses of 3 different microstructures of Ti-6Al-4V under static and dynamic conditions and different temperatures will be compared in this work. The heat treatments chosen are mill-annealing, $\alpha-\beta$ Annealing and $\beta$ Annealing. Johnson-Cook models will also be determined in conjunction with ABAQUS finite element software, which will finally be used to predict the behaviour of the different microstructures.

\section{Experimental methods}

\subsection{Material}

The material studied in this work is the $\alpha-\beta$ titanium alloy Ti-6Al-4V. The detailed chemical composition of the alloy is presented in Table 1 . Material is received in mill-annealing condition in a form of a $3 \mathrm{~mm}$ thickness plate. 
Table 1: Chemical composition of the Ti-6Al-4V alloy used

\begin{tabular}{|c|c|c|c|c|c|c|c|c|}
\hline & $\% \mathrm{Al}$ & $\% \mathrm{~V}$ & $\% \mathrm{Fe}$ & $\% \mathrm{C}$ & $\% \mathbf{H}$ & $\% 0$ & $\% \mathbf{N}$ & $\% \mathrm{Ti}$ \\
\hline At \% & 6.00 & 4.10 & 0.12 & 0.01 & 0.004 & 0.1 & 0.004 & Bal. \\
\hline
\end{tabular}

\subsection{Heat treatments}

With the aim of modifying the microstructure of the alloy, 3 different heat treatments (HTs) are considered, see Fig. 1:

- Mill-annealing (MA) (as-received): consists of two stages, (1) plastic deformation in the high-temperature $\alpha-\beta$ zone; (2) annealing at a lower temperature to relax residual stresses.

- $\quad \boldsymbol{\alpha}-\boldsymbol{\beta}$ annealing (ABA): heating occurs in the $\alpha-\beta$ region at a temperature of $940^{\circ} \mathrm{C}$ for one hour with air cooling and subsequent annealing against stress at $675^{\circ} \mathrm{C}$ for 4 hours. Aims at obtaining a $\alpha+\beta$ bimodal microstructure.

- $\quad \boldsymbol{\beta}$ Annealing (BA) heating up to $1025^{\circ} \mathrm{C}$, slightly higher than $\beta$-transus, for half an hour to avoid excessive beta grain growth, with air cooling. Subsequently, another heating is applied to $730^{\circ} \mathrm{C}$ for 2 hours. Aims at obtaining a completely laminar $\alpha$ phase structure.

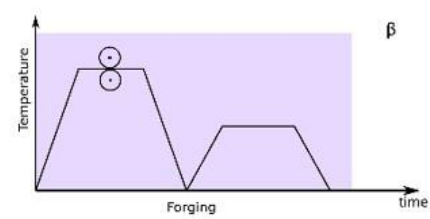

Mill-annealing

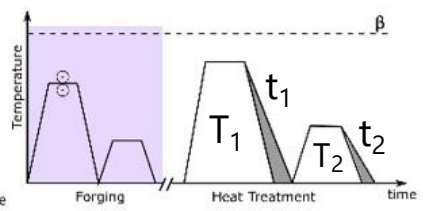

$\alpha+\beta$ annealing

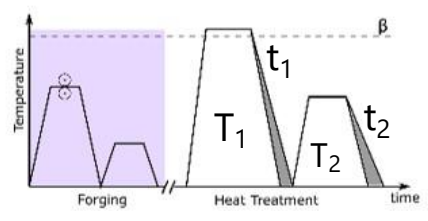

$\beta$ annealing

Figure 1: Diagram of the different heat-treatments used in this study.

Details of times and temperatures of the HTs are shown in Table 2.

Table 2: Details of the heat treatment considered in this study.

\begin{tabular}{rcccccc}
\hline Heat Treatment & $\mathbf{T}_{\mathbf{1}}\left({ }^{\circ} \mathbf{C}\right)$ & $\mathbf{t}_{\mathbf{1}}(\mathbf{h})$ & $\mathbf{C o o l i n g}$ & $\mathbf{T 2}\left({ }^{\circ} \mathbf{C}\right)$ & $\mathbf{t}_{\mathbf{2}}(\mathbf{h})$ & Cooling \\
\hline $\boldsymbol{\alpha}-\boldsymbol{\beta}$ annealing (ABA) & 940 & 1 & Air & 675 & 4 & Air \\
$\boldsymbol{\beta}$ annealing (BA) & 1025 & 0.5 & Air & 730 & 2 & Air \\
\hline
\end{tabular}

\subsection{Microscopy methods}

Scanning Electron Microscope (SEM) is used to characterise the microstructure and chemistry of the different material conditions. A Hitachi S-3400 SEM equipped with an Oxford Instruments energy dispersive X-ray (EDX) spectroscopy detector is used. Micrographs are obtained in back-scattered electrons (BSE) mode using $15 \mathrm{kV}$ accelerating voltage and $10 \mathrm{~mm}$ working distance. An optical microscope (Reichter microscope with Leica digital camera) is used to obtain low magnification micrographs. 


\subsection{Mechanical testing}

Dynamics and static mechanical tests are performed for the different heat treatments. A summary of the testing conditions is presented in Table 3.

Table 3: Testing conditions.

\begin{tabular}{rcc}
\hline Type of Test & Strain Rate $\left(\mathbf{s}^{\mathbf{- 1}}\right)$ & Temperature $\left({ }^{\circ} \mathbf{C}\right)$ \\
\hline Room-temperature Static & 0.001 & 25 \\
Room-temperature Dynamic & 500 & 25 \\
Mid-temperature Dynamic & 500 & 200 \\
High-temperature Dynamic & 500 & 400 \\
\hline
\end{tabular}

Two different geometries are used for the room temperature (RT) and the hightemperature (HT) conditions $\left(200^{\circ} \mathrm{C}, 400^{\circ} \mathrm{C}\right)$, see Fig. 2 . The HT samples present larger grip regions to increase the contact area with the electrodes that heat the specimens, as can be seen in Figure 3. The specific arrangements for each type of test are presented next.

Static \& Dynamic RT

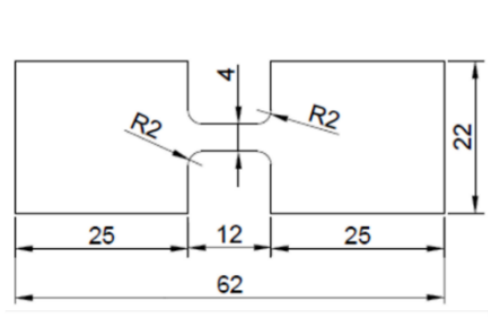

Dynamic HT

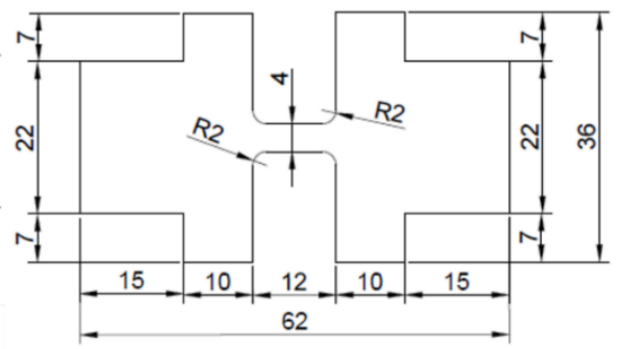

Figure 2: Geometry of the tensile specimens for RT and HT (thickness $=3 \mathrm{~mm}$ ).

Static tests: Static tests are performed at RT at a strain rate $10^{-3} \mathrm{~s}^{-1}$ in a servo-hydraulic MTS model 810 universal testing machine equipped with a $100 \mathrm{kN}$ load cell. An MTS model $632.25 \mathrm{C}-20$ extensometer with a calibrated base length of $50 \mathrm{~mm}$ was used to measure the strain during the test.

Dynamics tests: Dynamic tests are performed at different temperatures in a tensile split Hopkinson bar testing equipment designed and built at the Department of Materials Science of Tampere University of Technology [7]. The incident bar is made of a high strength steel (AISI 4340) and the transmitted bar is made of 2007 aluminum alloy. The specimens were fixed to the testing bars using the adhesive bonding methods detailed in Hueto et al. [6]. This fixing method present some practical disadvantages (more time consuming) but has the advantage of reducing electrical noise in the measurements. For HT tests, heating was carried out by means of the Joule effect caused by electric current passed through the specimen. The current is applied before the test through copper electrodes (see Fig. 2), being these released free just before the start of the test to avoid affecting the results. A thermocouple type $\mathrm{K}$ was welded to the samples to measure the temperature during the test. 


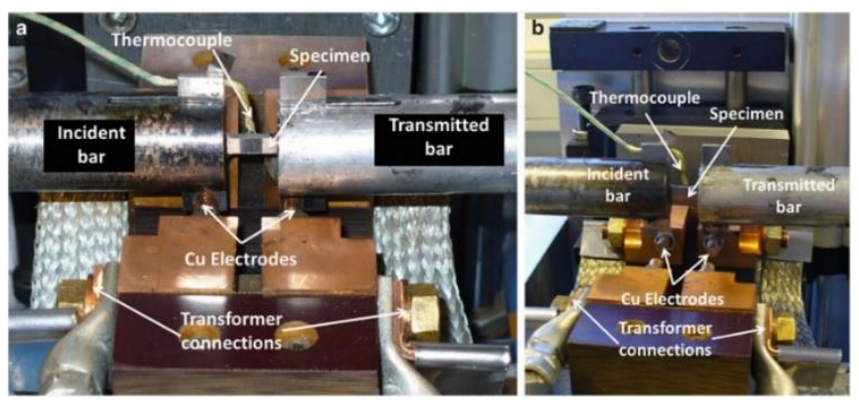

Figure 3: Copper electrode system for direct electric heating, Viscasillas [8].

\section{Results}

In this section, the results of the microstructural analysis are presented identifying the different microstructures relevant for Ti-6Al-4V components. Then, the mechanical behaviour of the alloy under different strain rates and temperatures is presented as a function of the microstructure.

\subsection{Microstructure analysis}

The different microstructures resulting from the three heat treatments considered in this study are presented in Fig. 4.

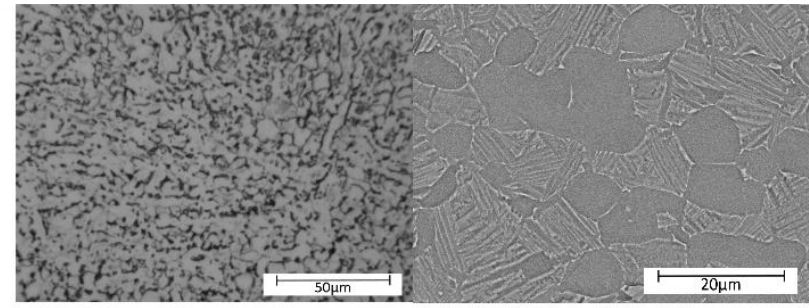

\section{Mill-annealing}

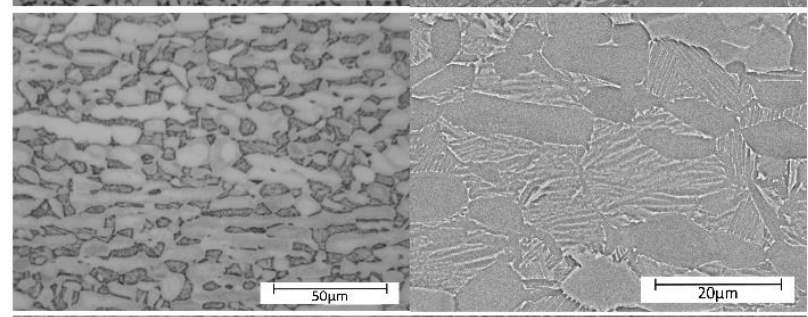

$\mathbf{a}+\boldsymbol{\beta}$ Annealing

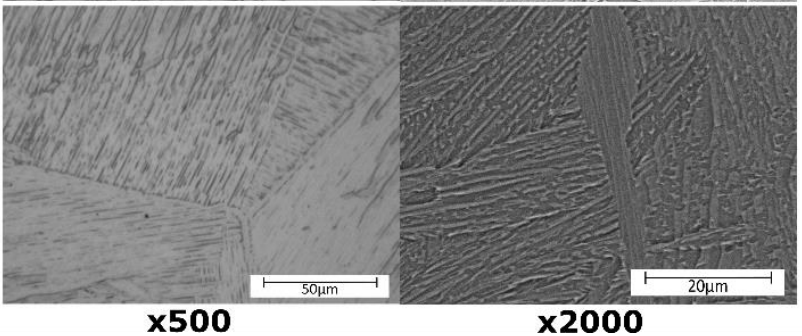

\section{B Annealing}

Figure 4: Optical (left) and SEM (right) Ti-6Al-4V microstructures resulting from the different HT considered in this study. 
For the mill-annealing condition, a combination of primary $\alpha$ grains inherited from the preannealing microstructure with $\alpha+\beta$ grains is observed. The percentage of both types of grains is about $50-50 \%$. A lamellar $\beta$ phase can be identified formed at the grain boundaries typical from this type of microstructures [9]. For the case of the $\alpha+\beta$ annealing the grain size of the primary $\alpha$ and the prior- $\beta$ grains is slightly increased respect to the mill-annealing conditions. The $\alpha$ lath thickness also show a slight increase. For the $\beta$ annealing, a fully $\alpha$ lamellar structure is found with a considerably larger prior- $\beta$ grain size than for the previous two cases. $\beta$ phase is observed to be formed at the $\alpha$ lath boundaries and the at the prior- $\beta$ grain boundaries. The thermo-mechanical behaviour of these microstructures under dynamics and static conditions are presented next.

\subsection{Mechanical behaviour of Ti-6AI-4V under extreme conditions}

The different microstructures detailed in the previous section were tested under dynamic and static conditions as stated in the methodology section. The mechanical behaviour of the different microstructures is presented in Fig. 5 in the form of stress-strain curves.

In terms of microstructural effects, for RT conditions there is a clear effect of the microstructure on the strength and more pronounced on the ductility of the alloy. In static, there is a slight increase of yield strength from the mill-annealing condition to both, $\alpha+\beta$ and $\beta$ annealing conditions in all the temperature regimes. The ductility drops in both static and dynamic conditions in the $\alpha+\beta$ and $\beta$ annealing states when compared to the mill-annealing condition, more abruptly for the $\beta$ annealing static case. This sudden drop of the ductility for the $\beta$ annealing case might be caused by the fully lamellar $\alpha$ structure causing a premature failure of the microstructure.

For the case of the effect of the testing conditions on the mechanical behaviour of the alloy, there is a clear decrease of the yield stress and strength of the material as the testing temperature increases for dynamic conditions. On the contrary, the ductility of the alloy shows an increase with temperature, especially pronounced in the $\beta$ and $\alpha+\beta$ annealing cases. These observations are in accordance with other studies in the literature [2,4]. More surprisingly, there is an increase of ductility from the static to dynamic condition at RT for the $\beta$ annealing case. These results are rationalised next using computational constitutive modelling of the alloy including the effect of microstructure.
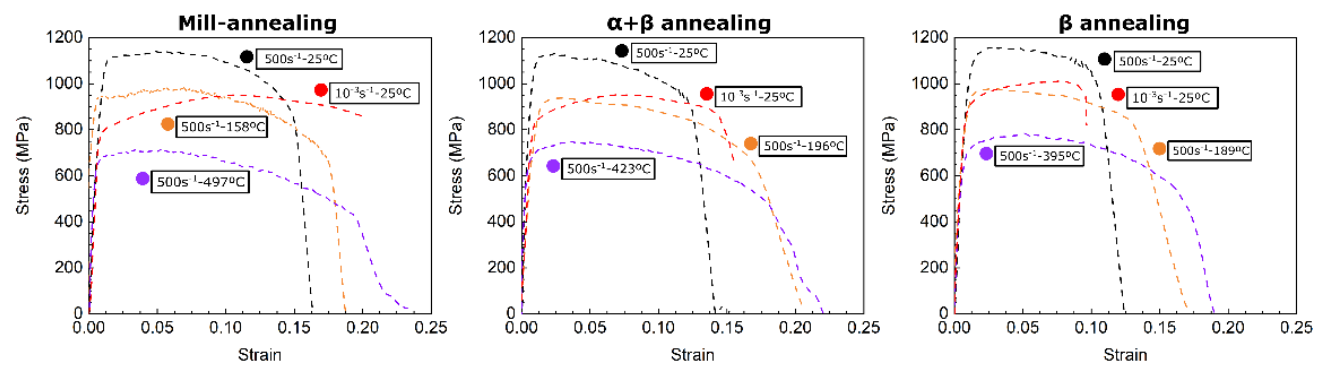

Figure 5: Experimentally observed behaviour of Ti-6Al-4V as a function of the temperature, strain rate and microstructure. 


\section{Discussion}

\subsection{Constitutive modelling of the dynamic behaviour of Ti-6Al-4V as a function of the microstructure}

Johnson \& Cook [10] proposed a model based on visco-plastic mechanics which defined the stress response in metals as a function of temperature, strain rate and strain hardening. Due to its versatility and dependence on a wide number of material and testing variables, it has been extensively used for dynamic problems in metallic alloys [11]. The flow stress $\bar{\sigma}$ in the Johnson-Cook (JC) model follows the equation

$$
\bar{\sigma}=\left[A+B\left(\bar{\varepsilon}^{p l}\right)^{n}\right]\left[1+C\left(\frac{\dot{\bar{\varepsilon}}^{p l}}{\dot{\varepsilon}_{0}}\right)\right]\left(1-T^{* m}\right)
$$

where $\bar{\varepsilon}^{p l}$ is the equivalent plastic strain, $\dot{\bar{\varepsilon}}^{p l}$ is the plastic strain rate, $\dot{\varepsilon}_{0}$ is the userdefined reference strain-rate and $A, B, C, n, m$ are material parameters. The nondimensional temperature $T^{*}$ is defined as $T^{*}=\left(T-T_{\text {room }}\right) /\left(T_{\text {melt }}-T_{\text {room }}\right)$, where $T_{\text {room }}$ is the RT and $T_{\text {melt }}$ is defined as the melting temperature of the alloy. Heat generated by plastic deformation is considered with the increase in temperature for a given element calculated as:

$$
\Delta T=\frac{\int \sigma d \bar{\varepsilon}^{p l}}{\rho C_{p}}
$$

where $C_{p}$ and $\rho$ are specific heat and mass density, respectively. For this study, these parameters are defined as $\rho=4.45 \mathrm{~g} / \mathrm{cm}^{3}$ and $C_{p}=530 \mathrm{~J} / \mathrm{kg} .{ }^{\circ} \mathrm{C}[12]$

In terms of damage, the model assumes that the equivalent plastic strain at the onset of damage is a function of stress triaxiality and strain rate. Is used for predicting the onset of damage due to the nucleation, growth, and coalescence of voids in ductile metals. The model follows the equation:

$$
\bar{\varepsilon}_{f}^{p l}=\left[d_{1}+d_{2} \exp \left(d_{3} \frac{p}{q}\right)\right]\left[1+d_{4} \ln \left(\frac{\dot{\bar{\varepsilon}}^{p l}}{\dot{\varepsilon}_{0}}\right)\right]\left(1-d_{5} T^{*}\right)
$$

where $\bar{\varepsilon}_{f}^{p l}$ is the strain at failure, p/q is a dimensionless pressure-deviatoric stress ratio ( $p$ is the pressure stress and $q$ is the Mises stress) and $d_{l}-d_{5}$ are material parameters.

This JC model is implemented in a 1D constitutive framework for calibration of the material parameters against the experimental curves in Fig. 5. The calibrated JC material parameters of the flow stress evolution for each microstructure are presented in Table 4. The calibrated JC damage parameters are presented in Table 5.

Table 4: JC material model parameters calibrated against experimental results

\begin{tabular}{cccccccc}
\hline & $\mathbf{A}(\mathbf{M P a})$ & $\mathbf{B}(\mathbf{M P a})$ & $\mathbf{C}$ & $\mathbf{n}$ & $\mathbf{m}$ & $\mathbf{T}_{\text {melt }}\left({ }^{\mathbf{o}} \mathbf{C}\right)$ & $\overline{\boldsymbol{\varepsilon}}_{\mathbf{0}}$ \\
\hline MA & 732 & 780 & 0.022 & 0.53 & 0.78 & 1653 & 0.001 \\
ABA & 800 & 700 & 0.020 & 0.60 & 0.75 & 1653 & 0.001 \\
BA & 880 & 780 & 0.015 & 0.65 & 0.79 & 1653 & 0.001 \\
\hline
\end{tabular}

Table 5: JC damage parameters calibrated against experimental results

\begin{tabular}{cccccc}
\hline & $\boldsymbol{d}_{\boldsymbol{1}}$ & $\boldsymbol{d}_{\boldsymbol{2}}$ & $\boldsymbol{d}_{\boldsymbol{3}}$ & $\boldsymbol{d}_{\boldsymbol{4}}$ & $\boldsymbol{d}_{\boldsymbol{5}}$ \\
\hline MA & 0.55 & 0.80 & 0.6 & -0.028 & 1.2 \\
ABA & 0.22 & 0.65 & 0.6 & -0.022 & 4.9 \\
BA & 0.11 & 0.20 & 0.6 & 0.030 & 4.9 \\
\hline
\end{tabular}


In order to study the effect of non-uniaxial stress states, local heating and necking the material model is implemented in FEA commercial software Abaqus. FEA models of the experimental tested specimens were set up simulating the experimental boundary conditions as shown in Fig. 6. The models are meshed with C3D10 elements with a total of 2000 elements for the whole model. For each testing condition, the exact temperature of the test extracted from the thermocouple welded to the samples was used.

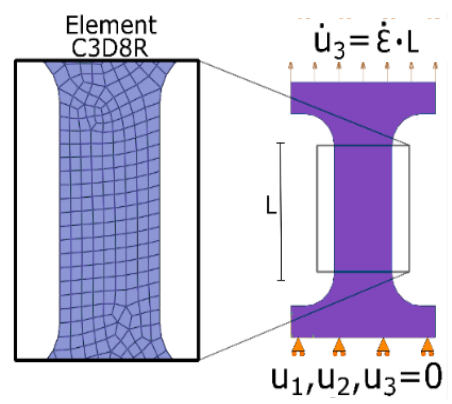

Figure 6: Mechanical boundary conditions in the FE simulation.

The computational results obtained from the 3D FE models are compared with the experimental behaviour in Fig. 7. The results show a good accordance with experimental measurements, both in the flow stress and in the damage behaviour. The initial hardening and the posterior adiabatic heating softening and necking is also captured by the computational models with good accuracy. In the following section, the specific case of ductility increase under dynamic conditions for the $\beta$ annealing case is rationalised using this model.
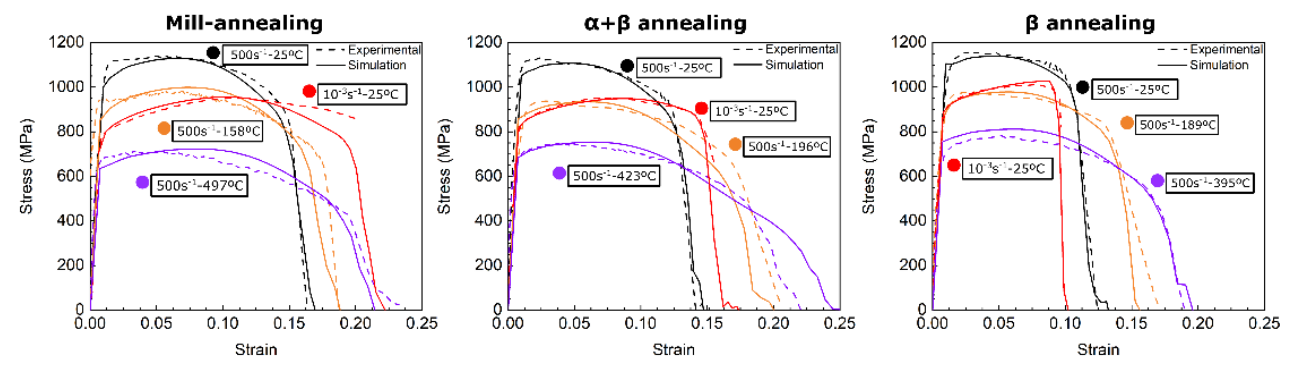

Figure 7 : Comparaison between experimental tests and FE simulation as a function of the temperature, strain rate and microstructure.

\subsection{Adiabatic heating and the on the thermomechanical behaviour of Ti-6Al-4V}

In the experimental results presented in Section 3.2, an abnormal increase of ductility from static to dynamic conditions is observed for the case of $\beta$ annealing. To further analyse this condition, the experimental and computational strain distributions are presented in Fig. 8, top. A good accordance between both strain distributions is observed. The temperature distribution from the simulations is shown in Fig. 8, bottom. A considerable local increase of $\sim 350^{\circ} \mathrm{C}$ is observed in the necking region. This is expected to be a main factor contributing to the ductility increase observed under dynamic conditions when compare to static tests. It can be inferred that this effect is dominant to this alloy when compared to the strain rate embrittlement usually observed in metals, as observed for the other two microstructures. 


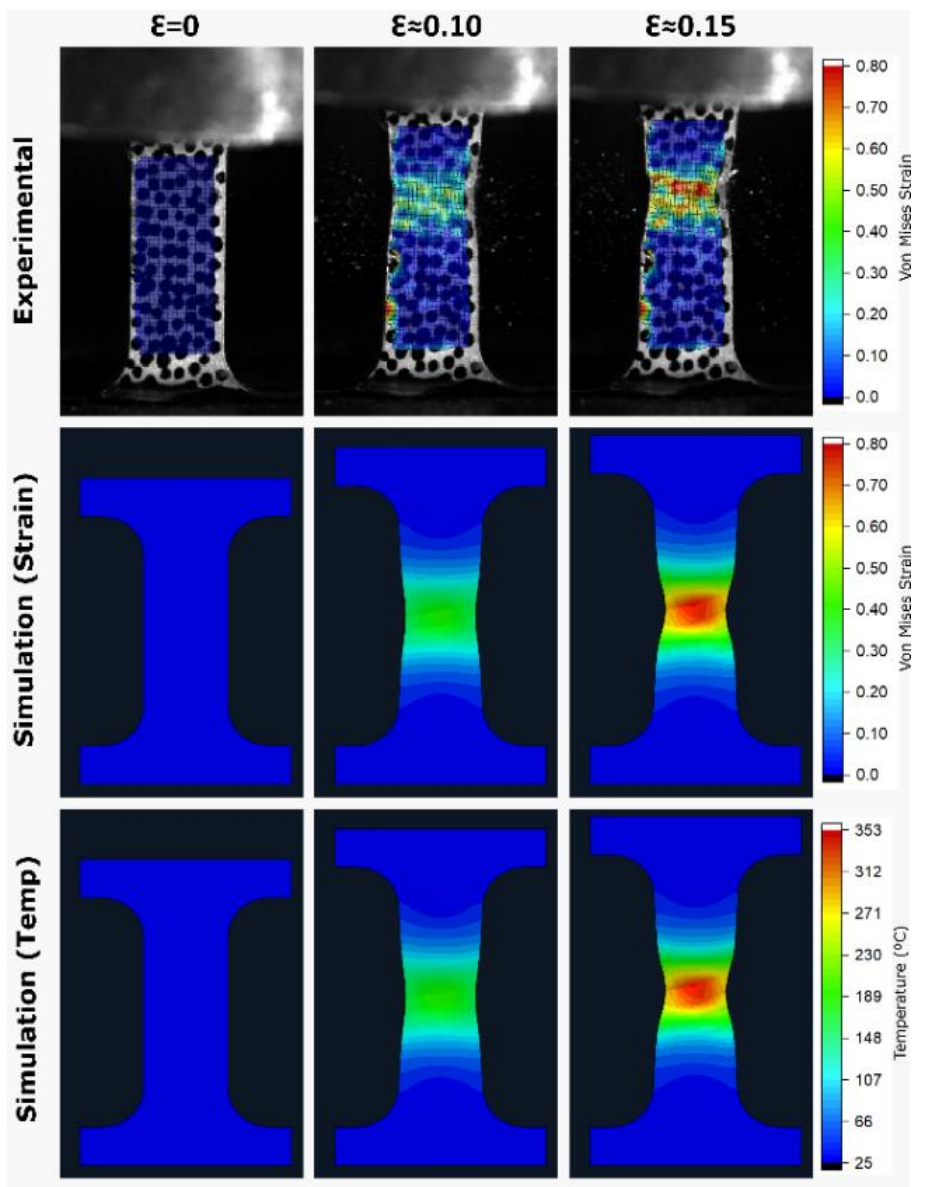

Figure 8: Experimental and computational strain distrubion for $\beta$-annealing dynamic test at RT and 500 $\mathrm{s}^{-1}$ (top) and the temperature distribution extracted from the simulated test (bottom).

\section{Conclusions}

The effect of the microstructure on the mechanical behaviour of Ti-6Al-4V under extreme conditions of temperature and strain rate has been studied in this work. From this study, the following conclusions can be drawn:

1. Three different microstructures are obtained using three different heattreatments: mill-annealing, $\alpha+\beta$ annealing and $\beta$ annealing.

2. For all the microstructures, there is an increase of ductility and a drop of the flow stress under dynamic conditions as the temperature increases.

3. For the $\beta$ annealing condition, there is an abnormal increase of ductility when dynamics conditions are used compared to static conditions. The computational results show that might be related to the effect of adiabatic heating and the strong temperature dependence of this microstructure.

4. A Johnson-Cook model is used to simulate the mechanical behaviour of the three microstructures. The model results capture with good accordance the evolution of the flow stress and the damage for all three microstructures. 


\section{References}

1. Royce, R. (2015). The jet engine. John Wiley \& Sons.

2. Lee, W. S., \& Lin, C. F. (1998). Plastic deformation and fracture behaviour of Ti-6Al$4 \mathrm{~V}$ alloy loaded with high strain rate under various temperatures. Materials Science and Engineering: A, 241(1-2), 48-59.

3. Lee, D. G., Lee, S., Lee, C. S., \& Hur, S. (2003). Effects of microstructural factors on quasi-static and dynamic deformation behaviors of Ti-6Al-4V alloys with widmanstätten structures. Metallurgical and Materials Transactions A, 34(11), 2541-2548.

4. Gangireddy, S., \& Mates, S. P. (2017). High temperature dynamic response of a Ti-6Al4V alloy: a modified constitutive model for gradual phase transformation. Journal of Dynamic Behavior of Materials, 3(4), 557-574.

5. Lin, Y. C., Wu, Q., Pang, G. D., Jiang, X. Y., \& He, D. G. (2020). Hot tensile deformation mechanism and dynamic softening behavior of Ti-6Al-4V alloy with thick lamellar microstructures. Advanced Engineering Materials, 22(3), 1901193.

6. Hueto, F., Hokka, M., Sancho, R., Rämö, J., Östman, K., Gálvez, F., \& Kuokkala, V. T. (2017). High temperature dynamic tension behavior of titanium tested with two different methods. Procedia engineering, 197, 130-139.

7. Hokka, M., Östman, K., Rämö, J., \& Kuokkala, V.-T. (2014). High Temperature Tension HSB Device Based on Direct Electrical Heating. En Dynamic Behavior of Materials, Volume 1 (págs. 227-233).

8. Viscasillas, M.J. (2019). Comportamiento en tenacidad de fractura y propagación de grietas en función del tratamiento térmico en aleaciones de titanio de aplicación aeroespacial. Tesis (Doctoral), E.T.S.I.A.E(UPM).

9. Lütjering, G., \& Williams, J. C. (2007). Titanium. Springer Science \& Business Media.

10. Johnson, G. R. (1983). A constitutive model and data for materials subjected to large strains, high strain rates, and high temperatures. Proc. 7th Inf. Sympo. Ballistics, 541547.

11. Shrot, A., \& Bäker, M. (2012). Determination of Johnson-Cook parameters from machining simulations. Computational Materials Science, 52(1), 298-304.

12. CES EduPack 2020. Version 20.1.1, Granta Design Limited. 